\title{
Tellurides with monovalent Ga and In - from chains to networks
}

\author{
T. Lindemann ${ }^{1}$, A. Isaeva ${ }^{2}$, O. Oeckler ${ }^{1}$ \\ ${ }^{1}$ Leipzig University, Faculty of Chemistry and Mineralogy, Leipzig, Germany, \\ ${ }^{2}$ University of Amsterdam, Department of Physics and Astronomy, Amsterdam, Netherlands \\ oliver.oeckler@gmx.de
}

\begin{abstract}
Monovalent inorganic gallium compounds are very rare, whereas this is not the case for indium compounds. As the chemistry of Ga(I) can be assumed to be dominated by its lone pair that favors unsymmetrical environments, the influence of the lone pair of $\operatorname{In}(\mathrm{I})$ is usually not very pronounced. Thus, there are very few isostructural Ga and In compounds. Starting from the elements, we have now obtained the new telluridogallates(I) $R E \mathrm{GaTe}_{2}$ and related compounds $R E \operatorname{InTe}_{2}(R E=\mathrm{La}-\mathrm{Nd})$. Although their orthorhombic unit-cell dimensions are similar, the structures combine modular entities in different ways that enable more or less space for lone pairs. In the case of In-containing compounds, data were collected using microfocused synchrotron radiation from microcrystals that were selected and pre-characterized by electron microscopy
\end{abstract}

The compounds $R E \mathrm{GaTe}_{2}$ crystallize in the non-centrosymmetric space group $P m c 2_{1}$, their lattice parameters reflect the lanthanide contraction. In contrast to telluridogallates(III) such as $\mathrm{CuGaTe}_{2}$ [1] and $\mathrm{AgGaTe}_{2}$ [2] that contain [GaTe 4 ] tetrahedral, its characteristical structural feature is a chain of $\mathrm{GaTe}_{3}$ pyramids sharing two Te atoms with neighboring pyramids. This would be typical for a telluridogallate(I), however, chemical bonding and charge distribution are not trivial. Bond valence sums confirm the electron-precise description according to $R E^{\mathrm{III}} \mathrm{Ga}^{\mathrm{I}} \mathrm{Te}^{-\mathrm{II}}$, and the coordination of gallium(I) is pyramidal as expected for a lone-pair atom. Bader charges for $\mathrm{LaGaTe}_{2}(\mathrm{La}+1.5, \mathrm{Ga}+0.5$, Te $-0.8--0.9)$ suggest only partial electron transfer. Thus, $R E \mathrm{GaTe}_{2}$ are rare examples of compounds with exclusively monovalent $\mathrm{Ga}$ atoms, probably the first one without organic residues. In $\mathrm{NdGaTe}_{2}$, a short Nd-Ga distance of $3.13 \AA$ is a possible indication of an interaction of the lone pair of $\mathrm{Ga}(\mathrm{I})$ with the $\mathrm{Nd}$ atoms. This is stronger than, for example, in $\mathrm{NdGaSb}_{2}(\mathrm{Nd}-\mathrm{Ga}$ distance $3.35 \AA$ ), [3] which has a different structure and bonding situation. Similar Nd-Ga distances are observed in intermetallic phases such as $\mathrm{NdGa}$ [4] or $\mathrm{NdGaRh}$ [5], so that a description as an oxidized intermetallic phase may also be considered. A formal consideration in the framework of the Zintl concept would assume a mixed chain-like $\left[\mathrm{Ga}^{(-2)} \mathrm{Te}^{(0)} \mathrm{Te}^{(-1)}\right]^{3-}$ polyanion; note that in these formal "charges" are note expected correspond to oxidation states. It is consistent with all descriptions that the distances of $2.67 \AA$ to the terminal Te atom are shorter than those to Te atoms bridging along the chain ( $2.95 \AA)$. Interactions between the polyanionic chains appear negligible. The Nd atoms are located in single-capped trigonal prisms of Te atoms, with $\mathrm{Ga}$ atoms forming two additional caps.

In contrast, compounds $R E \operatorname{InTe}_{2}$ are centrosymmetric, they adopt a structure with the space group Amm2. Although this can, in principle, be related to the structure of $R E \mathrm{GaTe}_{2}$ by group-subgroup relationships, the cationic modules are not shifted against each other in the indium compounds. The indium atoms are located in capped trigonal prisms that show little lone-pair influence, their environment is more symmetrical. Although these polyhedra are interconnected in a fashion that is similar to the one in $R E \mathrm{GaTe}_{2}$, the distances between the chains are not much larger than the ones within the chains so that the compound is a rare-earth indium (I) telluride rather than a telluridoindate(I) with a discrete polyanion. Still, bond valence sums correspond to $R E^{\mathrm{III}} \mathrm{In}^{\mathrm{I}} \mathrm{Te}^{\mathrm{II}}{ }_{2}$. The anionic Inor Ga-containing substructures thus show a pronounced influence on the arrangement of the cationic substructures - they interconnect similar modules is different ways. The CrB structure type may be regarded as an aristotype of both arrangements.

[1] T. Plirdpring, K. Kurosaki, A. Kosuga, T. Day, S. Firdosy, V. Ravi, G. J. Snyder, A. Harnwunggmoung, T. Sugahara, Y. Ohishi (2012) Adv. Mater. 24, 3622.

[2] S. Chatraphorn, T. Panmatarite, S. Pramatus, A. Prichavudhi, R. Kritayakirana, J.-O. Berananda, V. Sayakanit, J. C. Woolley (1985) J. Appl. Phys. 57, 1791.

[3] A. M. Mills, A. Mar, (2001) J. Am. Chem. Soc. 123, 1151.

[4] S. P. Yatsenko, A. A. Semyannikov, B. G. Semenov, K. A. Chuntonov (1979) J. Less-Common Met. 64, 185.

[5] F. Hulliger, (1996) J. Alloys Compd. 239, 131.

Keywords: monovalent gallium, lone pair effects, polyanions, modular structures

The authors thank the ESRF (Grenoble) for granting beamtime. 